\title{
ON THE COMPUTATIONAL TECHNIQUE OF OPTIMAL SYNTHESIS PROBLEM USING STRUCTURE PARAMETERS
}

\author{
NAONORI NISHIDA AND GARY J. POWERS \\ Department of Chemical Engineering, Carnegie-Mellon \\ University, Pittsburgh, Pennsylvania 15213 U. S. A.
}

\begin{abstract}
The process synthesis problem formulated by using structure parameters becomes, in general, a highly constrained nonlinear programming problem. This paper shows that by the use of a transformation in constrained structure parameters, however, all the constraints on the structure parameters are eliminated from the problem. This transformation makes it possible to ease significantly the computational difficulty of handling constrained structure parameters. Two computational methods are proposed, in which the incorporation of the transformation in constrained structure parameters is presented. The results of synthesis of two examples are presented and compared with other methods of synthesis. Computational experience with examples has indicated that different methods must be adopted depending on the type of problem.
\end{abstract}

\section{Introduction}

The problem of chemical processing systems synthesis is to determine both an optimal system structure and the optimal design variables of each process unit. The problem then becomes a combinatorial problem, in which the optimal arrangement of process units as well as their optimal value of design variables has to be determined. If the number of process units is large, the possible combinations become quite a large number. Therefore, to avoid solving the combinatorial problem directly, efficient methods have to be developed. However, if the number of process units is moderately large or small, the probable number of combinations may not become so large, if we can remove the unrealistic combinations from the possible ones by empirical experience or heuristics. Thus, in this case, we may find the optimal scheme with direct comparison of objectives of each scheme. We call this procedure discrete search hereafter.

One way of finding the optimal structure or optimal interconnection among process units is to transform the discrete nature of the synthesis problem into a continuous one. Ichikawa et al. ${ }^{4}$ have first proposed a so-called structure parameter approach, in which possible interconnections among process units are expressed by means of structure parameters. One prominent characteristic of using the structure parameters is that their introduction brings out a number

Received January 13, 1978 . Correspondence concerning this article should be addressed to N. Nishida, now with Department of Management Science, Science University of Tokyo, Tokyo 162. of constraints on them in the form not only of equality but also of inequality. Several constrained optimization techniques ${ }^{1,13)}$ may solve this problem. However, if we note the high dimensionality of structure parameters and their number of constraints, the optimization technique adopted has to be carefully chosen.

In this paper, computational methods are proposed for the structure parameter approach for synthesis of optimal process scheme. A transformation in constrained structure parameters is used to ease the computational difficulty of handling the constraints on the structure parameter. Two illustrative examples taken from previous workers ${ }^{5,6)}$ are solved to see how the proposed methods work.

\section{Formulation of Synthesis Problem}

The structure parameter approach to the optimal synthesis of process systems was introduced in several previous papers ${ }^{4,8-10)}$, and thus only a brief summary is described here.

Consider a steady-state process system consisting of $N$ process units or subsystems, whose input and output behaviour is expressed by

$$
g_{i}\left(\boldsymbol{x}_{i}, \boldsymbol{y}_{i}, \boldsymbol{d}_{i}\right)=0, \quad i=1,2, \cdots, N
$$

where $\boldsymbol{x}_{i}$ and $\boldsymbol{y}_{i}$ are, respectively, the input and output state vectors of the $i$-th subsystem of a process system; $\boldsymbol{d}_{i}$ the design vector. The structure of the process system is expressed by a set of structure parameters $\alpha_{i j}$ defined by

$$
\boldsymbol{x}_{i}=\sum_{j=1}^{N} \alpha_{i j} \boldsymbol{y}_{j} \quad i=1,2, \cdots, N
$$


The structure parameters have the following characteristics:

$$
\begin{aligned}
& \text { Equality constraint: } \sum_{i=1}^{N} \alpha_{i j}=1, j=1,2, \cdots, N \\
& \text { Inequality constraint: } 0 \leq \alpha_{i j} \leq 1 \\
& \qquad i, j=1, \cdots, N
\end{aligned}
$$

The following objective function for a system is defined as a sum of each subsystem objective function.

$$
J(\boldsymbol{x}, \boldsymbol{y}, \boldsymbol{\alpha}, \boldsymbol{d})=\sum_{i=1}^{N} J_{i}=\sum_{i=1}^{N} f_{i}\left(\boldsymbol{x}_{i}, \boldsymbol{y}_{i}, \boldsymbol{d}_{i}\right)
$$

With the above description of the process system, the synthesis problem under consideration can be defined as: "Find the optimal values of structure parameters and design variables which satisfy the subsystem equation, Eq. (1), to minimize the system objective function, Eq. (4), subject to the constraints, Eqs. (2) and (3)."

\section{The Use of Transformation in Constrained Struc- ture Parameters}

A typical characteristic of the structure parameter approach is the presence of a large number of constraints associated with structure parameters. As the synthesis problem of chemical engineering interest involves, in general, a number of nonlinear elements in the subsystem equations and/or the objective function, then it becomes a nonlinear constrained programming problem. The convergence rate of nonlinear constrained optimization is usually slow, and also the optimization step often terminates at a point where one or two constraints are merged, even when the current point is far from the optimum.

The approach adopted here is to transform the structure parameters and leave the objective function unaltered. To eliminate the equality constraint, Eq. (3-1), Nishida et $a l^{8}{ }^{8}$ have proposed the following transformation of the constraints.

$$
\left.\begin{array}{l}
\alpha_{1 i}=\zeta_{1 i} \\
\alpha_{2 i}=\zeta_{2 i}\left(1-\zeta_{1 i}\right) \\
\vdots \quad \vdots \\
\alpha_{j i}=\zeta_{j i} \prod_{k=1}^{j-1}\left(1-\zeta_{k i}\right) \\
\alpha_{N-2, i}=\zeta_{N-2, i} \prod_{k=1}^{N-3}\left(1-\zeta_{k i}\right), \quad i=1,2, \cdots, N-1
\end{array}\right\}
$$

from which $\alpha_{N-1, i}$ can be determined dependently as follows:

$$
\alpha_{N-1, i}=1-\sum_{j=1}^{N-2} \alpha_{j i} \quad i=1,2, \cdots, N-1
$$

Using $\boldsymbol{\zeta}$ instead of $\boldsymbol{\alpha}$ as the decision variables, the problem is transformed into determining the optimal values of $\zeta_{i j}$ subject to inequality constraint, Eq. (6).

It is well known that the following transformation restricts $\zeta$ to the range $0 \leq \boldsymbol{\zeta} \leq 1$.

$$
\zeta=\sin ^{2} \beta
$$

This transformation, however, has produced the periodicity of optimal solution in $\boldsymbol{\beta}$-space. Therefore, the optimization technique which will be adopted has to be a method which will confine the step-size within the primitive periods of function $\sin ^{2} \beta, \pi$.

\section{Computational Methods}

In the previous section, the use of transformations of constrained structure parameters made it possible to eliminate both inequality and equality constraints associated with structure parameters. However, many problems of actual chemical engineering interest involve a number of inequality constraints, both explicit and implicit, in the design variables. Then the synthesis problem becomes a constrained nonlinear programming problem in which a careful choice of optimization technique should be made.

The following two different methods are proposed.

\section{1 Simultaneous method}

The structure parameters and the design variables are updated at the same time, and the transformations of constrained structure parameters suggested in the previous section will be applied to the method. Umeda et al..$^{13)}$ and Mishra et al. ${ }^{6,7}$ ) have used the same simultaneous method in conjunction with the simplex pattern search technique ${ }^{2)}$, but the transformations of constrained structure parameters have not been utilized.

\section{2 Feasible two-level method}

This method consists of the first-level and the secondlevel problem. In the first level, the objective function of the system is minimized with respect to design variables with a given set of structure parameters. In the second level, the structure parameters are updated to minimize the same objective function.

\section{Illustrative Examples}

Two synthesis problems which have been used to illustrate how the present methods work are briefly described.

\section{1 Reactor-separator system synthesis ${ }^{5 \text { ) }}$}

The first synthesis problem was to determine an optimal system structure of a reactor-separator system, in which two types of reactors, continuous stirred tank reactor (CSTR) and plug flow reactor (PFR), and an ideal separator (SEP) are available as processing units. The reaction system which will take place in CSTR and/or PFR is the first-order irreversible reaction $A \stackrel{K}{\rightarrow} B$. The ideal separator (SEP) is assumed to separate $A$ and $B$ from their mixture. The inputoutput transformations and cost functions of the processing units along with the numerical values of the 


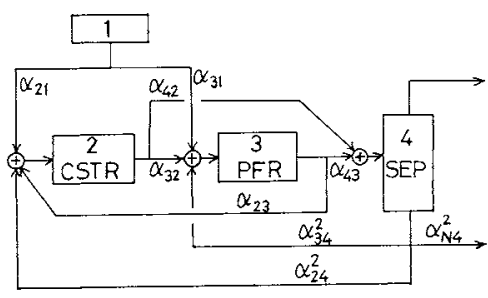

Fig. 1 System structure for synthesis of an optimal reactor-separator system

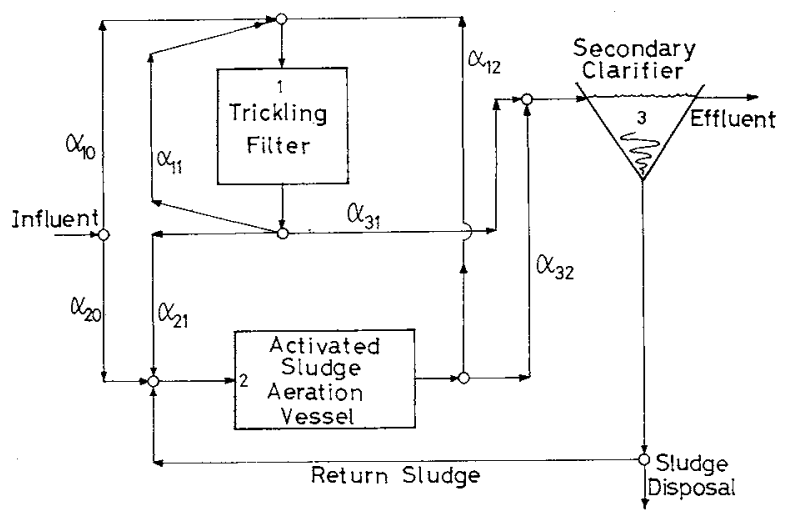

Fig. 2 System structure for synthesis of an optimal wastewater treatment system

parameters can be found in the paper of Osakada and Fan $^{11}$. With this definition of the system, the system synthesis problem is to determine the optimal system structure and the optimal design variables which minimize the following objective function:

$$
J=\sum_{i=1}^{N+1} f_{i}
$$

An integrated system structure which will be used to find the optimal structure parameters and the design variables is shown in Fig. 1. The equality and inequality constraints on the structure parameters are

$$
\left.\begin{array}{c}
\alpha_{10}+\alpha_{20}=1 \\
\alpha_{32}+\alpha_{42}=1 \\
\alpha_{23}+\alpha_{43}=1 \\
\alpha_{24}^{2}+\alpha_{34}^{2}+\alpha_{N 4}^{2}=1 \\
0 \leq \alpha_{10}, \alpha_{20}, \alpha_{32}, \alpha_{42}, \alpha_{23}, \alpha_{43}, \alpha_{24}^{2}, \alpha_{34}^{2}, \alpha_{N 4}^{2} \leq 1
\end{array}\right\}
$$

\section{2 Wastewater treatment system synthesis}

The second system example considered was to determine the optimal structure of a biological wastewater treatment system, composed of a trickling filter, an activated sludge aeration vessel, and a secondary clarifier. The problem was taken from Mishra et $\mathrm{l}^{6,7}$. It represents a typical system of nonlinearity and is rather complex compared to the previous example. The specific form of the material balance equations along with the numerical values of the parameters can be found as Eqs. (10) to (32) in the paper of Mishra et $a l^{6}{ }^{6}$. An integrated structure of this wastewater treatment system which has been used by Mishra et al. is shown in Fig. 2. The constraints on the structure parameters are

$$
\begin{gathered}
\alpha_{10}+\alpha_{20}=1 \\
\alpha_{11}+\alpha_{21}+\alpha_{31}=1 \\
\alpha_{12}+\alpha_{32}=1 \\
0 \leq \alpha_{10}, \alpha_{20}, \alpha_{11}, \alpha_{21}, \alpha_{31}, \alpha_{12}, \alpha_{32} \leq 1
\end{gathered}
$$

The objective function of the system is to minimize the total construction cost of the system, which consists of the cost of the trickling filter, $C^{T F}$, the aeration vessel, $C^{A S S}$, and the secondary clarifier, $C^{S C}$. They are expressed as

$$
J=1.4\left(C^{T F}+C^{A S}+C^{S C}\right)
$$

\section{Computed Results of Illustrative Examples}

\section{1 Reactor-separator system}

$\alpha_{12}, \alpha_{32}, \alpha_{23}, \alpha_{N 4}^{2}$, and $\alpha_{34}^{2}$ were chosen as the independent variable. In terms of transformation in the structure parameters, they are expressed as follows: $\alpha_{21}=\sin ^{2} \beta_{21}, \alpha_{32}=\sin ^{2} \beta_{32}, \alpha_{23}=\sin ^{2} \beta_{23}, \alpha_{N 4}^{2}=\sin ^{2} \beta_{N 4}^{2}$, and $\alpha_{34}^{2}=\sin ^{2} \beta_{34}^{2}\left(1-\sin ^{2} \beta_{N 4}^{2}\right)$. Then $\alpha_{31}, \alpha_{34}, \alpha_{43}$ and $\alpha_{24}^{2}$ are determined dependently as $\alpha_{31}=1-\alpha_{21}, \alpha_{42}=1$ $-\alpha_{32}, \quad \alpha_{43}=1-\alpha_{23}, \quad \alpha_{24}^{2}=1-\alpha_{N 4}^{2}-\alpha_{34}^{2} . \quad$ The design variables of this problem are the holding time of both CSTR and PFR, that is, $\theta_{2}$ and $\theta_{3}$. In this problem, no constraint is imposed on the design and state variables except the trivial non-negativeness of $\theta_{2}$ and $\theta_{3}$. Therefore, the optimization technique to be adopted here would be a class of gradient method such as the steepest gradient and conjugate gradient method. They are employed in order to compare their effectiveness.

Computed results for the two-level method are shown in Table 1. The computation time required for the conjugate gradient method was about three seconds by an IBM 360/67, compared to five seconds for the steepest gradient method. Table 1 shows that the optimal scheme consists of PFR and SEP with a recycle.

Computed results for the simultaneous method are shown in Fig. 3. The initial estimate of the independent variables is the same as that used for the twolevel approach. Using the conjugate gradient method required 24 iterations, where in every 8 iterations, the conjugate gradient routine was restarted. The final values of the objective function, structure parameters and the design variables are $J=-0.78212, \alpha_{21}=0.0$, $\alpha_{31}=1.0, \alpha_{32}=0.6367, \alpha_{42}=0.3633, \alpha_{23}=0.0, \alpha_{43}=1.0$, $\alpha_{N 4}^{2}=0.0, \quad \alpha_{34}^{2}=1.0, \quad \alpha_{24}^{2}=0.0, \theta_{2}=0.0191$, and $\theta_{3}=$ 0.0780 , and the corresponding final values of transformed structure parameters are $\beta_{21}=-0.00106$, $\beta_{32}=0.92381, \beta_{23}=0.00088, \alpha_{N 4}^{2}=0.00002$, and $\alpha_{34}^{2}=$ 1.56696 , where $\beta_{21}, \beta_{32}, \beta_{23}, \beta_{N^{4}}^{2}$, and $\beta_{34}^{2}$ are expressed in radians. The computation time required was 
Table 1 Computed results of two-level method for reactorseparator system synthesis

\begin{tabular}{|c|c|c|c|c|c|c|}
\hline Iteration & $J$ & $\alpha_{19}$ & $\alpha_{43}$ & $\alpha_{34}^{2}$ & $\theta_{2}$ & $\theta_{3}$ \\
\hline \multicolumn{7}{|c|}{ (Steepest gradient method) } \\
\hline 0 & -0.75199 & 0.5000 & 0.5000 & 0.5000 & 0.0176 & 0.0505 \\
\hline 1 & -0.77897 & 0.1052 & 0.9603 & 0.9620 & 0.0114 & 0.0757 \\
\hline 2 & -0.78159 & 0.0241 & 0.9960 & 0.9967 & 0.0152 & 0.0776 \\
\hline 3 & -0.78195 & 0.0066 & 0.9989 & 0.9996 & 0.0019 & 0.0779 \\
\hline 4 & -0.78211 & 0.0004 & 0.9998 & 0.9999 & 0.0011 & 0.0779 \\
\hline 5 & -0.78212 & 0.0000 & 1.0000 & 0.9999 & 0.0012 & 0.0779 \\
\hline \multicolumn{7}{|c|}{ (Conjugate gradient method) } \\
\hline 0 & -0.75199 & 0.5000 & 0.5000 & 0.5000 & 0.0176 & 0.0505 \\
\hline 1 & -0.77897 & 0.1052 & 0.9603 & 0.9620 & 0.0114 & 0.0757 \\
\hline 2 & -0.78211 & 0.0002 & 1.0000 & 0.9987 & 0.0069 & 0.0779 \\
\hline 3 & -0.78212 & 0.0000 & 1.0000 & 1.0000 & 0.0069 & 0.0779 \\
\hline
\end{tabular}

about five seconds. While the convergence rate of the conjugate gradient method was favourable, 100 iterations were consumed for the steepest gradient method. This slow convergence may be due to the probable existence of a highly asymmetric curved valley on the $\theta_{2}-\theta_{3}$ plane of the contours of the objective function.

In the computation of the simultaneous approach, the initial value of $\theta_{2}$ and $\theta_{3}$ was set in such a way that with an initial set of structure parameters, the optimization on $\theta_{2}$ and $\theta_{3}$ was carried out, and thus these values obtained were used as the initial estimate of $\theta_{2}$ and $\theta_{3}$. In doing so, favourable convergence was attained. However, for a case where the initial value of $\theta_{2}$ and $\theta_{3}$ was set at an arbitrary number, such as $\theta_{2}=\theta_{3}=0.1$, over 100 iterations were required even when the method of the conjugate gradient was employed.

Ichikawa and $F_{a n}{ }^{51}$, Osakada and $F^{111}{ }^{11}$, and Stephanopoulos et $a l{ }^{12)}$ have found the same scheme as the present one. Osakada et al. have used an infeasible two-level technique in conjunction with structure parameter approach. Over 200 iterations were required to solve the solution of the same problem by their algorithm. Another infeasible twolevel method, into which Hestene's method of multipliers was incorporated has been shown by Stephanopoulos et al. to be quite feasible as a computational tool. Their method, however, required 90 iterations to solve the same problem. In the present feasible two-level method, the use of transformation of constrained structure parameters has made it possible to reduce significantly the number of iterations required for the second level. The periodicity of optimal solutions in $\beta_{i j}$ space has not caused any numerical problem.

\section{2 Biological wastewater treatment system}

$\alpha_{10}, \alpha_{11}, \alpha_{21}$ and $\alpha_{32}$ were chosen as the independent structure parameters. Their transformation forms are given as: $\alpha_{10}=\sin ^{2} \beta_{10}, \alpha_{11}=\sin ^{2} \beta_{11}, \alpha_{21}=\sin ^{2} \beta_{21}$ $\left(1-\sin ^{2} \beta_{11}\right), \alpha_{32}=\sin ^{2} \beta_{32}$, from which $\alpha_{20}, \alpha_{31}$ and $\alpha_{12}$ are determined by $\alpha_{20}=1-\alpha_{10}, \alpha_{31}=1-\alpha_{11}-\alpha_{21}$, and

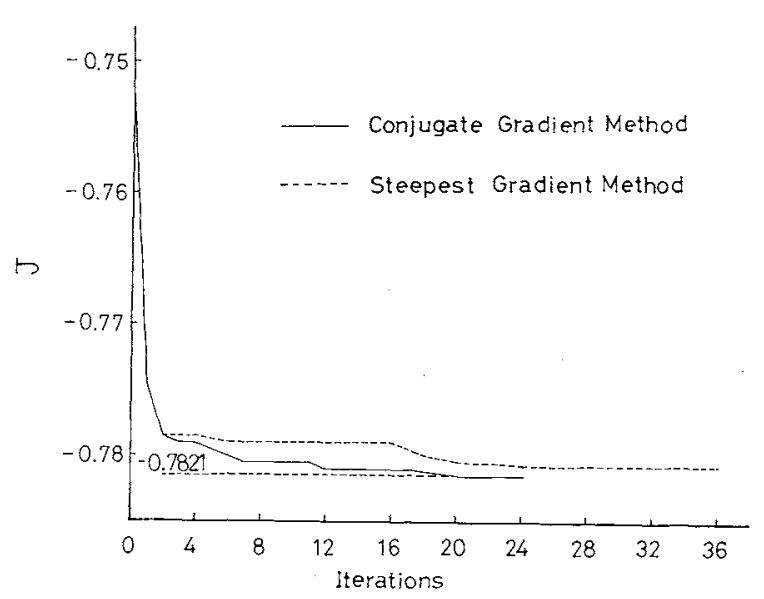

Fig. 3 Comparison of gradient methods for synthesis of an optimal reactor-separator system

$\alpha_{12}=1-\alpha_{32} . \quad x_{1}^{1}, x_{1}^{2}, x_{2}^{2}$ and $r$ were chosen as the other independent variables. Since recycle ratio $r$ is constrained between one and zero, the transformation $r=\sin ^{2} \beta_{r}$ is used. This problem involves several inequality constraints which appear in the independent variables implicitly. Therefore, non-gradient method would be suitable for the optimization technique, and thus the simultaneous search was conducted by using the Complex method of $\mathrm{Box}^{2}$. The two-level method was also tested. However, it performed poorly. The reason is that in the first level of the two-level method the program for finding the optimal variables often terminates on the undesirable local minimum so that guadratic approximation along the gradient direction failed to find the true minimum.

Preliminary computation has revealed that the recycle ratio $r$ of the optimized structure tended to be a small value, so that the concentration of cells in sludge recycle stream of the optimized structure reached the possible upper limit of $1 \times 10^{6} \mathrm{ppm}$. Obviously, this result cannot be implemented in a practical process. From an engineering point of view, the concentration of cells in the sludge recycle stream should be less than some appropriate value. Mishra et al., have not imposed the constraint on the upper value of the 
Table 2 Computed results of discrete search for Case $1, x_{2}^{r} \leq 1 \times 10^{5}$

\begin{tabular}{|c|c|c|c|c|c|c|c|}
\hline$k$ & Scheme & Cost $[\$]$ & $D^{r F}$ & $A^{S G}$ & $V^{A S}$ & $w$ & $r$ \\
\hline $\begin{array}{l}2.4 \\
{\left[\text { day }^{-1}\right]}\end{array}$ & $\begin{array}{l}1 \\
2 \\
3 \\
4\end{array}$ & $\begin{array}{l}168,076 \\
173,315 \\
168,217 \\
657,702\end{array}$ & $\begin{array}{l}0.0 \\
0.0 \\
0.00009 \\
19.126\end{array}$ & $\begin{array}{l}5,073 \\
5,061 \\
5,063 \\
2,363\end{array}$ & $\begin{array}{l}0.09558 \\
0.09626 \\
0.09650 \\
6.2 \times 10^{-15}\end{array}$ & $\begin{array}{l}1.02 \times 10^{-4} \\
1.04 \times 10^{-4} \\
9.17 \times 10^{-5} \\
3.64 \times 10^{-3}\end{array}$ & $\begin{array}{l}0.0854 \\
0.0841 \\
0.0899 \\
0.4344\end{array}$ \\
\hline $\begin{array}{l}0.48 \\
{\left[\text { day }^{-1}\right]}\end{array}$ & $\begin{array}{l}1 \\
2 \\
3 \\
4\end{array}$ & $\begin{array}{l}242,169 \\
247,999 \\
242,557 \\
657,762\end{array}$ & $\begin{array}{l}0.0 \\
0.0 \\
0.00009 \\
19.178\end{array}$ & $\begin{array}{l}8,241 \\
8,262 \\
8,057 \\
2,286\end{array}$ & $\begin{array}{l}0.1572 \\
0.1568 \\
0.1699 \\
3.6 \times 10^{-14}\end{array}$ & $\begin{array}{l}1.66 \times 10^{-8} \\
6.59 \times 10^{-7} \\
6.40 \times 10^{-8} \\
4.47 \times 10^{-3}\end{array}$ & $\begin{array}{l}0.1487 \\
0.1500 \\
0.1370 \\
0.5944\end{array}$ \\
\hline $\begin{array}{l}0.24 \\
{\left[\mathrm{day}^{-1}\right]}\end{array}$ & $\begin{array}{l}1 \\
2 \\
3 \\
4\end{array}$ & $\begin{array}{l}\text { Infeasible } \\
457,389 \\
425,763 \\
657,819\end{array}$ & $\begin{array}{r}2.989 \\
2.878 \\
19.179\end{array}$ & $\begin{array}{r}11,128 \\
11,172 \\
2,284\end{array}$ & $\begin{array}{l}0.2241 \\
0.1858 \\
1.6 \times 10^{-13}\end{array}$ & $\begin{array}{l}3.23 \times 10^{-8} \\
5.83 \times 10^{-8} \\
4.43 \times 10^{-3}\end{array}$ & $\begin{array}{l}0.1189 \\
0.1450 \\
0.3563\end{array}$ \\
\hline
\end{tabular}

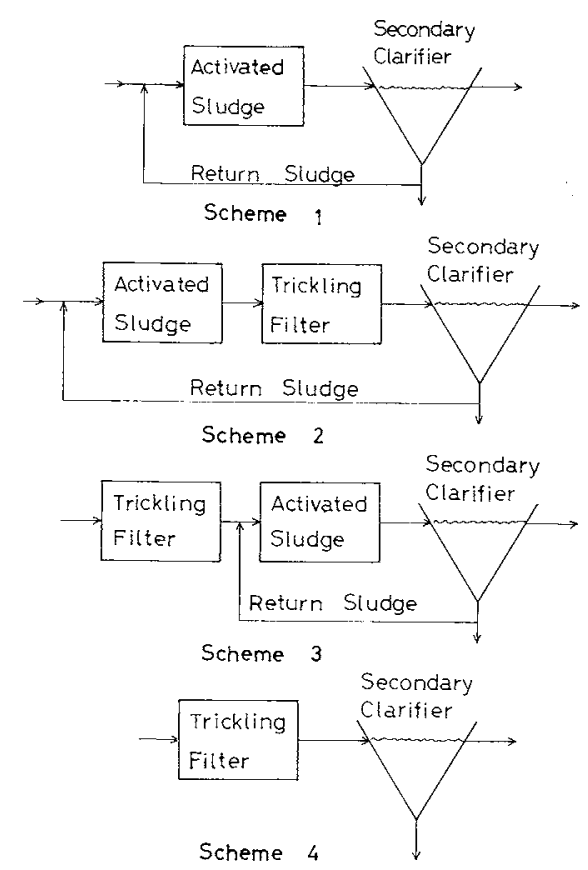

Fig. 4 Alternate schemes for synthesis of wastewater treatment system

recycle sludge concentration, although their resulting values of the recycle sludge concentration were far from the possible upper limit of $1 \times 10^{6} \mathrm{ppm}$. Himmelblau ${ }^{3}$ ? has solved the same problem by using the Generalized Reduced Gradient Method ${ }^{1)}$ and has imposed the upper bound on the recycle sludge concentration with $1 \times 10^{5} \mathrm{ppm}$. This value of $1 \times 10^{5} \mathrm{ppm}$ is still high, as the case may be. With different values of the upper bound on the recycle sludge concentration, two cases were investigated. For Case $1,1 \times 10^{5} \mathrm{ppm}$ was imposed as the upper bound of the recycle sludge concentration so that the present result could be compared with that of Himmelblau as well as Mishra et al. For Case 2, the upper bound of $0.3 \times 10^{5} \mathrm{ppm}$ was used for a practical case.

1) Results of discrete search: Four system schemes were taken into consideration for screening the system performance of the possible combinations of trickling filter, activated sludge aeration vessel and secondary clarifier. These schemes are shown in Fig. 4. In this discrete search, $x_{1}^{1}, x_{1}^{2}, x_{2}^{2}$ and $r$ are chosen as decision variable to find the optimal system of the restricted structure. Table 2 shows the results of the optimal design of four schemes for Case 1.

For $k=2.4$ and $k=0.48$, Table 2 shows that the best system is Scheme 1, regardless of the constraint of the return sludge concentration $x_{2}^{r}$. Although the cost of Schemes 2 and 3 is slightly higher than that of Scheme 1, the system of Schemes 2 and 3 is essentially the same as that of Scheme 1, since the trickling filter vanishes in Schemes 2 and 3. In both Cases 1 and 2, the cost of Scheme 2 was observed to be higher by about $\$ 5,200$ to $\$ 6,400$ than that of Scheme 1. This difference is attributed mainly to the difference in the cost of recycle pumping, and partly to the difference in the values of the optimized independent variables.

For $k=0.24$, Scheme 3 becomes the most costeffective system. Note that Scheme 1, which has been the best scheme for $k=2.4$ and $k=0.48$, is no longer capable of satisfying the specified quality of effluent to be discharged because of low value of the maximum specific growth rate, $k$, of microbes in the activated sludge aeration vessel. This results in a change in system configuration. The characteristic of these results is that the concentration of the return sludge should be maintained as high as possible, so that the recycle ratio $r$ is minimized.

2) Results of continuous search: Computed results of continuous search are shown in Table 3. The initial values of the vertices for the independent variables in the Complex method were generated as pseudo-random numbers. For example, for $k=2.4$ and $k=0.48$ the pseudo-random numbers were generated in the following interval:

$$
\begin{aligned}
& 0 \leq x_{1}^{1} \leq 90,6 \leq x_{1}^{2} \leq 60,10,000 \leq x_{2}^{2} \leq 50,000, \\
& 0.4 \leq \beta_{10}, \beta_{11}, \beta_{21}, \beta_{32}, \beta_{r} \leq 1.2
\end{aligned}
$$

The number of iterations required in the Complex method ranged from 269 to 325 . The computation time required ranged from four to five seconds for an IBM 360/67. In most cases, the convergence be- 
Table 3 Computed results of continuous search for wastewater system

\begin{tabular}{|c|c|c|c|c|c|c|}
\hline \multirow[t]{2}{*}{ Variable } & \multicolumn{3}{|c|}{ Case 1. $x_{2}^{r} \leq 1 \times 10^{5} \mathrm{ppm}$} & \multicolumn{3}{|c|}{ Case 2. $x_{2}^{r} \leq 0.3 \times 10^{5} \mathrm{ppm}$} \\
\hline & $k=2.4$ & $k=0.48$ & $k=0.24$ & $k=2.4$ & $k=0.48$ & $k=0.24$ \\
\hline Total cost $[\$]$ & 168,132 & 242,551 & 426,670 & 182,776 & 283,742 & 479,787 \\
\hline$\alpha_{10}$ & 0.0006 & 0.0267 & 0.9865 & 0.0 & 0.0045 & 0.9994 \\
\hline$\alpha_{20}$ & 0.9994 & 0.9733 & 0.0135 & 1.0 & 0.9955 & 0.0006 \\
\hline$\alpha_{11}$ & 0.6108 & 0.2962 & 0.0069 & 0.3049 & 0.6893 & 0.0006 \\
\hline$\alpha_{21}$ & 0.3056 & 0.6991 & 0.9929 & 0.5756 & 0.3077 & 0.9994 \\
\hline$\alpha_{31}$ & 0.0836 & 0.0047 & 0.0002 & 0.1195 & 0.0030 & 0.0 \\
\hline$\alpha_{12}$ & 0.0011 & 0.0258 & 0.0030 & 0.0 & 0.0088 & 0.0011 \\
\hline$\alpha_{32}$ & 0.9989 & 0.9742 & 0.9970 & 1.0 & 0.9912 & 0.9989 \\
\hline$D^{T F}[\mathrm{ft}]$ & 0.0008 & 0.0049 & 2.823 & 0.0 & 0.0 & 3.502 \\
\hline$A^{S O}\left[\mathrm{ft}^{2}\right]$ & 5,074 & 8,240 & 11,336 & 5,116 & 8,563 & 10,689 \\
\hline$V^{A S}[\mathrm{MG}]$ & 0.0956 & 0.1574 & 0.1867 & 0.1406 & 0.2828 & 0.3310 \\
\hline$w[--]$ & $1.0 \times 10^{-4}$ & $2.8 \times 10^{-8}$ & $5.4 \times 10^{-8}$ & $4.5 \times 10^{-4}$ & $1.7 \times 10^{-7}$ & $6.3 \times 10^{-8}$ \\
\hline$r[-]$ & 0.0849 & 0.1440 & 0.1449 & 0.1830 & 0.3112 & 0.2986 \\
\hline$x_{1}^{\mathrm{I}}[\mathrm{ppm}]$ & 38.341 & 54.680 & 77.063 & 44.093 & 38.560 & 72.685 \\
\hline$x_{1}^{2}[\mathrm{ppm}]$ & 8.257 & 15.014 & 18.518 & 9.431 & 15.023 & 17.719 \\
\hline$x_{2}^{2}[\mathrm{ppm}]$ & 7,860 & 12,923 & 12,652 & 4,668 & 7,190 & 6,907 \\
\hline$\beta_{10}$ & 0.0243 & 0.1640 & 1.4544 & 0.0016 & 0.0670 & 1.5947 \\
\hline$\beta_{11}$ & 0.8971 & 0.5755 & -0.0834 & 0.5846 & 0.9791 & -0.0239 \\
\hline$\beta_{21}$ & 2.0528 & 1.4890 & 1.5585 & 1.1433 & 1.6691 & 1.5727 \\
\hline$\beta_{32}$ & 1.6042 & 1.7321 & 1.5161 & 1.5734 & 1.4767 & 1.6036 \\
\hline$\beta_{r}$ & 0.2956 & 0.3892 & 0.3905 & 0.4419 & 0.5917 & 0.5781 \\
\hline
\end{tabular}

haviour was quite favourable. To illustrate the fast convergence of the present method, the values of every twentieth iteration for $J, \alpha_{10}, \alpha_{32}$ and $r$ in Case 2, where $k=2.4$, are shown in Table 4. For $k=2.4$ and $k=0.48$, the resulting system structure consists of an activated sludge aeration vessel and a secondary clarifier in series. This system structure is the same as the best system obtained by discrete search, which is shown in Scheme 1 of Fig. 4. We can thus conclude here that for $k=2.4$ and $k=0.48$ the optimal system consists of an activated sludge aeration vessel and a secondary clarifier in series, and no stream splitting is allowed in any structure parameter.

A further decrease in $k$ to a value of 0.24 led to a system structure in which all feed sewage goes to the trickling filter, all effluent of the trickling filter goes to the activated sludge aeration vessel and all aeration vessel effluent goes to the secondary clarifier. This system structure is shown in Scheme 3 of Fig. 4. This system is the same as the best system obtained in the discrete search.

It is interesting to compare the present results with those of Mishra et $a l^{6,7}$ and of Himmelblau ${ }^{3}$. Mishra et al. have first proposed a problem of the biological wastewater treatment system synthesis. For the optimal synthesis of the system they utilized a simplex pattern search technique in conjunction with structure parameter approach, in which no use of transformation in the constrained structure parameters is reported. For $k=2.4$, the optimal system structure obtained by Mishra et al., is essentially the same as that by the present method, although the total cost of $\$ 219,766$ obtained with their simultaneous search is much higher than the present cost values of
Table 4 Convergence behaviour of continuous search for the wastewater treatment system synthesis

\begin{tabular}{rrrrr}
$\begin{array}{c}\text { No. of } \\
\text { iterations }\end{array}$ & $J[\$]$ & $\alpha_{10}$ & $\alpha_{32}$ & $r$ \\
\hline 1 & $1,660,073$ & 0.7176 & 0.7683 & 0.6863 \\
21 & 362,561 & 0.2275 & 0.8063 & 0.6737 \\
61 & 262,580 & 0.0785 & 0.9473 & 0.4395 \\
81 & 219,189 & 0.0478 & 0.9889 & 0.1598 \\
101 & 194,615 & 0.0068 & 0.9917 & 0.2630 \\
121 & 186,710 & 0.0000 & 0.9999 & 0.1807 \\
272 & 183,895 & 0.0000 & 0.9999 & 0.1863 \\
\hline
\end{tabular}

$\$ 168,132$ for Case 1 and $\$ 182,776$ for Case 2. For $k=0.48$ the optimal system structure of Mishra et al. is quite different from the present structure. Their system structure involves both a trickling filter and an activated sludge system, with the influent wastewater being split approximately equally between the two process systems. And their total cost of $\$ 550,061$ is again much higher than the cost of the present result. For $k=0.24$ their optimal system consists of trickling filter, aeration vessel and secondary clarifier, with about $70 \%$ of the trickling filter effluent going to the aeration vessel and the remaining $30 \%$ to the secondary clarifier. This system structure with a cost of $\$ 699,884$ is again different from the present one, where no stream splitting occurs in the trickling filter effluent and the total cost is $\$ 426,670$ for Case 1 and $\$ 479,787$ for Case 2.

Himmleblau ${ }^{3)}$ has solved the same problem of wastewater treatment system synthesis proposed by Mishra et al. With the Generalized Reduced Gradient Method $^{1 \text {, }}$ Himmelblau found the same system structure for $k=0.48$ as the one obtained by the present method. The total cost of Himmelblau's system, 
$\$ 243,841$, with an upper bound constraint on the recycle sludge concentration of $1 \times 10^{5} \mathrm{ppm}$ is slightly higher than that of $\$ 242,551$ for the present system.

\section{Discussion and Conclusion}

The structure parameter approach is one way of solving a process synthesis problem. Introducing structure parameters, however, brings out a number of constraints on them in the form of both equality and inequality. The use of transformation in constrained structure parameter, however, has made it possible to remove constraints on the structure parameter. Even though such constraints can be eliminated from the problem in terms of transformation, the problem may still have constraints on the design and/or state variables. Therefore, the optimization technique to be adopted should be carefully chosen depending on the problem with or without design and/or state variable constraints as well as problem size.

For a small-size problem without design and/or state variable constraints such as the reactor-separator synthesis problem ${ }^{3}$ ) both the two-level and the simultaneous method proposed here would work successfully with gradient methods. For a small-size problem with design and/or state variable constraints such as the wastewater treatment system synthesis, the simultaneous method would be better compared with the two-level method. One of the optimization codes to be incorporated into the simultaneous method would be derivative free optimization methods such as the Complex method of Box. Other optimization codes with derivatives such as the Generalized Reduced Gradient Method of Abadie et al. ${ }^{11}$ also work favourably, as has been reported by Himmelblau ${ }^{3)}$. However, the penalty function method may perform poorly ${ }^{3}$.

Decomposition technique may be one possible way to solve the structure parameter synthesis problem. To ease the difficulty of computations of structure optimization problem, several authors ${ }^{11-13)}$ have proposed decomposition techniques and their methods have been tested in a small-size problem of the reactorseparator synthesis problem. Even for this smallsize problem, a great number of iterations were required to obtain its optimal structure, as they have reported. Experience with this sample problem indicates that decomposition is not an effective technique.

For a large-size problem, no conclusion about the method to be adopted can be drawn, since no computational experience with a large-size example has been gained. The exploitation of this powerful approach to large-size problems will be left to future work.

\section{Nomenclature}

$A \quad=$ substance $A$

$A^{S C} \quad=$ cross-sectional area of secondary clarifier $\quad\left[\mathrm{ft}^{2}\right]$

$B \quad=$ substance $B$ [-]

$C^{A S} \quad=$ construction cost of aeration vessel

$C^{S C} \quad=$ construction cost of secondary clarifier

$C^{T^{T}}=$ construction cost of trickling filter $\quad$ [\$]

$c_{i} \quad=$ cost factor $\quad[-]$

$d \quad=$ design vector $\quad[-]$

$D^{T F} \quad=$ depth of trickling filter [ft]

$f_{i} \quad=$ performance function of subsystem $i \quad[-]$

$g_{i} \quad=i$-th subsystem equation defined by Eq. (1) $\quad[-]$

$J \quad=$ performance function of system [-]

$K \quad=$ reaction rate constant $[-]$

$k=$ maximum specific growth rate of cells $\left[\right.$ day $\left.^{-1}\right]$

$r \quad=$ sludge recycle ratio $\quad[-]$

$V^{A S} \quad=$ aeration vessel volume $\quad[\mathrm{MG}]$

$\boldsymbol{w} \quad=$ sludge wasting ratio

$x \quad=$ input stream state vector $\quad[-]$

$x_{1}^{1}=$ concentration of organic nutrients at aeration filter exit

[ppm]

$x_{1}^{2}=$ concentration of organic nutrients at trickling vessel exit

[ppm]

$\begin{array}{llr}x_{2}^{2} & =\text { concentration of cells in effluent } & \text { [ppm] } \\ y & =\text { output stream state vector } & {[-]}\end{array}$

$\alpha \quad=$ structure parameters $[-]$

$\beta \quad=\begin{aligned} & \text { transformed structure parameters } \\ & \text { defined by Eq. (8) [-] }\end{aligned}$

$\zeta \quad=\underset{\text { transformed structure parameters }}{\text { defined by Eq. }(5)}$

$\theta \quad=$ holding time of reactor $[-]$

\section{Literature Cited}

1) Abadie, J. and J. Guigou: "Gradient Reduit Generalisé," Note HI 069/01, Electricité de France, Clamant, France, April (1969).

2) Box, M. J.: The Computer Journal, 8, 42 (1966).

3) Himmelblau, D. M.: paper presented at U. S.-Japan Seminar, July (1975).

4) Ichikawa, A., N. Nishida, and T. Umeda: Preprints of 34th Annual Meeting of The Soc. of Chem. Engrs., Japan (1969).

5) Ichikawa, A., and L. T. Fan: Chem. Eng. Sci., 28, 357 (1973).

6) Mishra, P. N., L. T. Fan, and L. E. Erickson: Can. J. Chem. Eng., 51, 694 (1973).

7) Mishra, P. N., L. T. Fan, and L. E. Erickson: ibid., 51, 702 (1973).

8) Nishida, N., A. Ichikawa, and E. Tazaki: Ind. Eng. Chem., Process Des. Dev. 13, 209 (1974).

9) Nishida, N., and A. Ichikawa: ibid., 14, 236 (1975).

10) Nishida, N., Y. A. Liu, and A. Ichikawa: $A I C h E J ., 22$, 539 (1976).

11) Osakada, K., and L. T. Fan: Can. J. Chem. Eng., 51, 94 (1973).

12) Stephanopoulos, G., and A. W. Wetserberg: ibid., 53, 551 (1975).

13) Umeda, T., A. Hirai, and A. Ichikawa: Chem. Eng. Sci., 27, 795 (1972).

14) Umeda, T., and A. Ichikawa: paper presented at AIChE Meeting, Dallas, Texas (1972). 